\title{
ON THE INFLUENCE OF TRANSITIVELY NORMAL SUBGROUPS ON THE STRUCTURE OF SOME INFINITE GROUPS
}

\author{
Leonid A. Kurdachenko and Javier Otal
}

\begin{abstract}
A transitively normal subgroup of a group $G$ is one that is normal in every subgroup in which it is subnormal. This concept is obviously related to the transitivity of normality because the latter holds in every subgroup of a group $G$ if and only if every subgroup of $G$ is transitively normal. In this paper we describe the structure of a group whose cyclic subgroups (or a part of them) are transitively normal.
\end{abstract}

2010 Mathematics Subject Classification: Primary: 20E15; Secondary: 20F16, 20F19.

Key words: Transitively normal subgroup, Dedekind group, $T$-group, $\bar{T}$-group, supersoluble group, hypercyclic group, radical group, generalized radical group.

\section{Introduction}

In the Theory of Groups, one of the topics that has received considerable attention since a long time is the study of the transitivity of normality or, more precisely, the influence on the structure of a group of that property. In the literature, a group $G$ is said to be a $T$-group if every subnormal subgroup of $G$ is normal, and therefore the mentioned question is the study of the structure of $T$-groups. Trivial examples of $T$-groups are abelian groups and groups whose subgroups are normal (Dedekind groups). Actually R. Dedekind [6] was able to determine the form of a finite non-abelian group with normal subgroups only, a result extended by R. Baer [1] to the general case. The structure of finite soluble $T$-groups is due to W. Gaschütz [8]. Since subgroups of $T$-groups need not be $T$-groups, a group $G$ is said to be a $\bar{T}$-group if every subgroup of $G$ is a $T$-group. Gaschütz himself proved that every finite soluble $T$-group is a $\bar{T}$-group although this is no longer true for infinite soluble groups. Infinite soluble $T$-groups and $\bar{T}$-groups were studied by D. J. S. Robinson $[\mathbf{2 2}]$. 
Recently L. A. Kurdachenko and I. Ya. Subbotin [14] introduced the concept of transitively normal subgroup. A subgroup $H$ of a group $G$ is said to be transitively normal in $G$ if $H$ is normal in every subgroup $K$ such that $H \leq K$ and $H$ is subnormal in $K$. There is an obvious relationship between transitivity of normality and transitively normal subgroups because a group $G$ is a $\bar{T}$-group if and only if every subgroup of $G$ is transitively normal. In the literature, there are many known types of subgroups that are transitively normal. We mention some of the most important of them.

- Pronormal subgroups. A subgroup $H$ of a group $G$ is said to be pronormal in $G$ if for each element $g \in G$ there exists some $u \in$ $\left\langle H, H^{g}\right\rangle$ such that $H^{g}=H^{u}$. P. Hall introduced pronormal subgroups and these subgroups appeared in a natural way in the process of investigation of significant subgroups of finite (soluble) groups as Sylow subgroups, Hall subgroups, system normalizers, and Carter subgroups. They also appeared in the study of soluble finite dimensional linear groups as well as in the study of many infinite soluble groups, where they usually are complements to the locally nilpotent residual. Clearly every pronormal subgroup is transitively normal, and in [14] some groups whose transitively normal subgroups are pronormal were considered.

- Weakly normal subgroups. These subgroups arose in the paper by K. H. Müller [19]. A subgroup $H$ of a group $G$ is said to be weakly normal in $G$ if $H^{g} \leq N_{G}(H)$ implies that $g \in N_{G}(H)$. Every pronormal subgroup is weakly normal and every weakly normal subgroup is transitively normal [2, Lemma 1 and Proposition 1].

- $\mathcal{H}$-subgroups. A subgroup $H \leq G$ is said to be an $\mathcal{H}$-subgroup of $G$ if $N_{G}(H) \cap H^{g} \leq H$ holds for every element $g \in G$ [4]. Every $\mathcal{H}$-subgroup is also transitively normal $[4$, Theorem 6$]$.

- NE-subgroups. Li Shirong [17] introduced the following type of $\mathcal{H}$-subgroup. A subgroup $H$ of a group $G$ is called an $N E$-subgroup if $N_{G}(H) \cap H^{G}=H$.

All these subgroups are transitively normal but not conversely. Thus, groups whose subgroups are $\mathcal{H}$-subgroups (respectively $N E$-subgroups, weakly normal subgroups, pronormal subgroups) are $\bar{T}$-groups. In this framework the following question naturally arises: is it possible to characterize the class of $\bar{T}$-groups by means of specific families of subgroups? For example, T. A. Peng [20] showed that a finite group is 
a $\bar{T}$-group if and only if its cyclic subgroups are pronormal. This result was extended to infinite locally soluble groups by I. Ya. Subbotin and N. F. Kuzenny $[\mathbf{2 5}$. P. Csörgö and M. Herzog proved that a finite group whose cyclic subgroups of prime odd order and of order 4 are $\mathcal{H}$-subgroups is supersoluble $([\mathbf{5}$, Theorems 8,10$])$. The same conclusion for a finite group whose analogous cyclic subgroups are $N E$-subgroups was obtained by Li Yangming ([18, Theorem 4.1]). In this paper we obtain the following generalization of these results for infinite locally finite groups.

Theorem A. Let $G$ be a locally finite group whose cyclic subgroups of prime order and of order 4 are transitively normal. Then $G$ is hypercyclic, the locally nilpotent residual $L$ of $G$ is an abelian Hall subgroup, and every subgroup of $L$ is $G$-invariant.

We recall that the locally nilpotent residual $L$ of a group $G$ is the intersection of all normal subgroups $H$ of $G$ such that $G / H$ is locally nilpotent. It is not hard to see that if $G$ is a locally finite group, then $G / L$ is locally nilpotent. We also recall that a group $G$ is said to be hypercyclic if $G$ has an ascending series of normal subgroups whose factors are cyclic while $G$ is said to be hypercentral if $G$ has an ascending central series. Hypercyclic groups (respectively, hypercentral groups) are the infinite analogs of supersoluble groups (respectively, nilpotent groups).

We note that in general the locally nilpotent residual $L$ has no complement. For example, there exist periodic hypercyclic groups $G$ whose locally nilpotent residual $L$ is abelian, every subgroup of $L$ is $G$-invariant, $G / L$ is abelian, but $L$ has no complement (see [11, $\S 2$, Example 1], [12, Theorem 4.7]). For finite groups the situation is different since $L$ is complemented by Schur-Zassenhaus Theorem.

We now list some wide characterizations of $\bar{T}$-groups that appear in the literature. Recall that a subgroup $H$ of a group $G$ is said to be primary if $H$ is a $p$-group for some prime $p$.

- A finite group whose cyclic subgroups are pronormal is a $\bar{T}$-group. (T. A. Peng $[\mathbf{2 0}]$ ).

- A locally finite group whose cyclic subgroups are pronormal is a $\bar{T}$-group. (I. Ya. Subbotin and N. F. Kuzennyu [25]).

- A finite group whose subgroups are $\mathcal{H}$-subgroups is a $\bar{T}$-group. (M. Bianchi, A. G. B. Mauri, M. Herzog, and L. Verardi [4]).

- A finite group whose primary subgroups are $\mathcal{H}$-subgroups is a $\bar{T}$ group. (M. Bianchi, A. G. B. Mauri, M. Herzog, and L. Verardi [4]). 
- A finite group whose subgroups are NE-subgroups is a $\bar{T}$-group. (Li Yangming [18]).

- A finite group whose primary subgroups are NE-subgroups is a $\bar{T}$ group. (Li Yangming [18]).

Making use of Theorem A, we are able to obtain the following most wide characterization of locally finite $\bar{T}$-groups.

Corollary A1. A locally finite group $G$ whose primary cyclic subgroups are transitively normal is a $\bar{T}$-group.

In Section 3, we study non-periodic groups whose cyclic subgroups are transitively normal. To avoid trivial cases, we consider non-abelian groups that satisfy a wide generalized solubility condition. A group $G$ is said to be radical if it has an ascending series whose factors are locally nilpotent (see [21]). $G$ is said to be generalized radical if $G$ has an ascending series whose factors are locally nilpotent or locally finite. Since the subgroup generated by all locally nilpotent normal subgroups of a group $G$ is locally nilpotent (the locally nilpotent radical of $G$ ) and the subgroup generated by all locally finite normal subgroups is locally finite (the locally finite radical of $G$ ), a generalized radical group has an ascending series of normal subgroups whose factors are either locally nilpotent or locally finite. We also note that a periodic generalized radical group is locally finite, and hence a periodic locally generalized radical group is also locally finite.

Theorem B. Let $G$ be a non-periodic, non-abelian, locally generalized, radical group whose cyclic subgroups are transitively normal. Then there exists an abelian normal subgroup $L$ of $G$ and an element $b \in G$ such that $G=L\langle b\rangle, b^{2} \in L$ and $x^{b}=x^{-1}$ for every element $x \in L$. Moreover

(1) if $b^{2}=1$, then the Sylow 2-subgroup $D$ of $L$ is elementary abelian; and

(2) if $b^{2} \neq 1$, then either $D$ is elementary abelian or $D=E \times\langle v\rangle$, where $E$ is elementary abelian and $\langle b, v\rangle$ is a quaternion group.

In both cases $\langle D, b\rangle$ is a Dedekind group. Conversely, if a group $G$ has such an structure, then every cyclic subgroup of $G$ is transitively normal.

It is worth mentioning that the results of Section 3 hold if we only impose that primary cyclic and infinite cyclic subgroups are transitively normal (though the proofs are more tedious). On the other hand it can be shown that non-periodic locally generalized radical groups whose cyclic subgroups are pronormal are abelian, and it is an open question to study other related properties for which the conditions of Theorem B are sufficient. 


\section{Auxiliary results}

In this section we establish properties of transitively normal subgroups to be frequently used in what follows. The first of them are almost immediate.

Lemma 1.1. Let $H$ be a transitively normal subgroup of a group $G$. If $L$ is a subgroup such that $H \leq L$, then $H$ is transitively normal in $L$.

Lemma 1.2. Let $H$ be an ascendant subgroup of a group $G$. If $H$ is transitively normal in $G$, then $H$ is normal in $G$.

This was shown in [14, Lemma 1.1].

The next results study locally nilpotent subgroups.

Lemma 1.3. Let $L$ be a locally nilpotent subgroup of a group $G$ and let $K$ be a finitely generated subgroup of $L$. If $K$ is transitively normal in $G$, then $K$ is normal in $L$. In particular, if $L$ is normal in $G$, then so is $K$.

Proof: Let $\mathcal{L}$ be the local system of $L$ consisting of all finitely generated subgroups of $L$ that contain $K$. If $F \in \mathcal{L}$, then $F$ is nilpotent since it is finitely generated. It follows that $K$ is subnormal in $F$ and hence normal in $F$ by Lemma 1.1. Since $L=\bigcup_{F \in \mathcal{L}} F, K$ is normal in $L$, as required. If $L$ is normal in $G$, then $K$ is subnormal in $G$ hence normal in $G$ since it is transitively normal.

As we mentioned in the Introduction, a Dedekind group $G$ is a group whose subgroups are all normal. R. Baer's characterization [1] of Dedekind groups showed that these groups $G$ are either abelian or can be expressed as a direct product $G=Q \times B \times D$, where $Q$ is a copy of the quaternion group of order $8, B$ is an elementary abelian 2-group and $D$ is a periodic abelian group with all elements of odd order.

Corollary 1.4. Let $L$ be a locally nilpotent subgroup of a group $G$. If every cyclic subgroup of $L$ is transitively normal in $G$, then $L$ is a Dedekind subgroup.

Proof: Since every cyclic subgroup of $L$ is normal in $L$ by Lemma 1.3, it follows that every subgroup of $L$ is normal in $L$.

Corollary 1.5. Let $L$ be a locally nilpotent ascendant subgroup of a group $G$. If every cyclic subgroup of $L$ is transitively normal in $G$, then every subgroup of $L$ is $G$-invariant.

Proof: By Lemma 1.2, $L$ is normal in $G$. Corollary 1.4 yields that $L$ is a Dedekind group. It follows that every cyclic subgroup of $L$ is subnormal in $G$. Being transitively normal, every cyclic subgroup of $L$ is $G$-invariant. Therefore, every subgroup of $L$ is $G$-invariant. 
The following results appear to be of a different nature but it will be useful in the study both of the periodic and non-periodic cases.

Lemma 1.6. Let $A$ be an elementary abelian normal p-subgroup of a group $G$, where $p$ is a prime. If every subgroup of $A$ is $G$-invariant, then the following conditions hold:

(1) If $g \notin C_{G}(A)$, then there exists a positive integer $k(g)$ such that $a^{g}=a^{k(g)}$ for every element $a \in A$; and

(2) $G / C_{G}(A)$ is a cyclic group of order dividing $p-1$.

Proof: For some set $\Lambda$, we express $A=\operatorname{Dr}_{\lambda \in \Lambda}\left\langle a_{\lambda}\right\rangle$, where the $a_{\lambda}$ have order $p$. Pick $g \in G$. For every $\lambda \in \Lambda$ the subgroup $\left\langle a_{\lambda}\right\rangle$ is $G$-invariant and then there exists some $p^{\prime}$-number $k(g, \lambda)$ such that $a_{\lambda}^{g}=a_{\lambda}^{k(g, \lambda)}$. Let $C_{\lambda}$ be an isomorphic copy of the group $U(\mathbb{Z} / p \mathbb{Z})$ of the units of the prime field $\mathbb{Z} / p \mathbb{Z}$ and think of every $k(g, \lambda) \in \mathbb{Z} / p \mathbb{Z}$ in the obvious way. Then we have a map

$$
\varphi: G \longrightarrow \mathrm{Cr}_{\lambda \in \Lambda} C_{\lambda} \text { given by } g \mapsto(k(g, \lambda))_{\lambda \in \Lambda},
$$

which is not hard to see that is a homomorphism.

We claim that all the numbers appearing in $\varphi(g)$ are equal (modulo $p$ ). Pick $\lambda, \mu \in \Lambda$ a couple of indices. Then we have

$$
a_{\lambda}^{g}=a_{\lambda}^{k(g, \lambda)}
$$

and

$$
a_{\mu}^{g}=a_{\mu}^{k(g, \mu)},
$$

and so

$$
\left(a_{\lambda} a_{\mu}\right)^{g}=a_{\lambda}^{g} a_{\mu}^{g}=a_{\lambda}^{k(g, \lambda)} a_{\mu}^{k(g, \mu)} .
$$

On the other hand, since $\left\langle a_{\lambda} a_{\mu}\right\rangle$ is $G$-invariant, there must exist some $d$ such that

$$
\left(a_{\lambda} a_{\mu}\right)^{g}=\left(a_{\lambda} a_{\mu}\right)^{d}=a_{\lambda}^{d} a_{\mu}^{d} .
$$

From this it readily follows that

$$
k(g, \lambda) \equiv d \equiv k(g, \mu) \quad(\bmod p),
$$

and then our claim follows.

Then the mapping $\kappa: G \longrightarrow U(\mathbb{Z} / p \mathbb{Z})$ given by $\kappa(g)=k(g, \lambda)$ the common number, is an epimorphism of $G$ onto $U(\mathbb{Z} / p \mathbb{Z})$. Since Ker $\kappa=$ $C_{G}(A)$, the result follows. 


\section{Locally finite groups whose cyclic subgroups are transitively normal}

As we mentioned in the Introduction, the results of P. Csörgö and M. Herzog [5] and Li Yangming [18] focused on finite groups whose cyclic subgroups of order 4 and $p \neq 2$ are $\mathcal{H}$-subgroups and $N E$-subgroups respectively and showed that such a group $G$ is supersoluble. We impose here the condition that such subgroups are transitively normal to obtain the same conclusion and extend the result to locally finite groups.

If $G$ is a group, $\Pi(G)$ is the set of all primes $p$ such that $G$ has elements of order $p$.

Lemma 2.1. A finite group $G$ whose cyclic subgroups of prime order and of order 4 are transitively normal is supersoluble.

Proof: Let $D$ be a Sylow 2-subgroup of $G$ and put $T=N_{G}(D)$. Since the cyclic subgroups of $D$ of order 2 and 4 are transitively normal in $T$, they are normal in $T$. By [16, Theorem 1], the Hall 2'-subgroup $K$ of $G$ is normal and then we have a semidirect decomposition $G=K 入 D$.

Let $p \in \Pi(K)$ and $P$ be a Sylow $p$-subgroup of $K$. Being transitively normal, every subgroup of $P$ of order $p$ is normal in $N_{G}(P)$. By [16, Theorem 2], $G$ is supersoluble.

Corollary 2.2. If the cyclic subgroups of prime order and of order 4 of a locally finite group $G$ are transitively normal, then $G$ has a unique Hall 2'-subgroup that is normal in $G$.

Proof: Let $\mathcal{L}$ be the local system of $G$ consisting of the finite subgroups of $G$. By Lemma 2.1, every $L \in \mathcal{L}$ is supersoluble and then $L$ has a unique Hall $2^{\prime}$-subgroup that have to be normal in $L$. Therefore $G$ has a unique Hall 2 -subgroup that is normal in $G$.

We deduce immediately some features of the groups of this section.

Corollary 2.3. If the cyclic subgroups of prime order and of order 4 of a locally finite group $G$ are transitively normal, then the commutator subgroup $[G, G]$ is locally nilpotent. In particular, the locally nilpotent residual $L$ of $G$ is locally nilpotent.

Proof: Let $\mathcal{L}$ be a local system of $G$ consisting of all finite subgroups. If $M \in \mathcal{L}$, by Lemma 1.1 and Lemma 2.1, $M$ is supersoluble. It follows that $[M, M]$ is nilpotent. Clearly

$$
[G, G]=\bigcup_{M \in \mathcal{L}}[M, M],
$$

and then $[G, G]$ is locally nilpotent. Clearly $L \leq[G, G]$. 
Corollary 2.4. Let $L$ be the locally nilpotent residual of a locally finite $G$. If the cyclic subgroups of prime order and of order 4 are transitively normal in $G$, then $2 \notin \Pi(L)$.

Proof: Let $D$ be a Hall $2^{\prime}$-subgroup of $G$. By Corollary $2.2, D$ is normal in $G$. Then the factor-group $G / D$ is a locally finite 2 -group and, in particular, it is locally nilpotent. It follows that $L \leq D$, as required.

Let $p$ be a prime and let $G$ be a $p$-group. For every integer $n \geq 1$, let

$$
\Omega_{n}(G)=\left\langle x \in G \mid x^{p^{n}}=1\right\rangle .
$$

If $p$ is odd, then $G$ is said to be $p$-central if $\Omega_{1}(G) \leq \zeta(G)$. If $p=2$, then $G$ is said to be 2 -central if $\Omega_{2}(G) \leq \zeta(G)$. To establish some results needed to state the main ones of this section, we first need a result by T. J. Laffey [15, Corollary 1 to Lemma 1].

Lemma 2.5. Let $G$ be a finite p-group, where $p$ is a prime. Suppose that $G$ is $p$-central. If $p$ is odd, then $\Omega_{n+1}(G) / \Omega_{n}(G) \leq \zeta\left(G / \Omega_{n}(G)\right)$ for each integer $n \geq 1$. If $p=2$, then $\Omega_{2 n+2}(G) / \Omega_{2 n}(G) \leq \zeta\left(G / \Omega_{2 n}(G)\right)$ for each integer $n \geq 1$.

Corollary 2.6. Let $G$ be a locally finite $p$-group, where $p$ is a prime. Suppose that $G$ is p-central. If $p$ is odd, then $\Omega_{n+1}(G) / \Omega_{n}(G) \leq \zeta\left(G / \Omega_{n}(G)\right)$ for each integer $n \geq 1$. If $p=2$, then $\Omega_{2 n+2}(G) / \Omega_{2 n}(G) \leq \zeta\left(G / \Omega_{2 n}(G)\right)$ for each integer $n \geq 1$.

Lemma 2.7. Let $G$ be a locally finite group and $P$ be a normal $p$-subgroup of $G$, where $p$ is an odd prime. Suppose that every subgroup of $P$ of prime order is transitively normal in $G$. If $G \neq C_{G}\left(\Omega_{1}(P)\right)$, then the following conditions hold:

(1) $L_{1}=\Omega_{1}(P)$ is an elementary abelian p-subgroup;

(2) $\Omega_{n+1}(G) / \Omega_{n}(G) \leq \zeta\left(C_{G}\left(L_{1}\right) / \Omega_{n}(G)\right.$ ) for each integer $n \geq 1$ (in particular, $P$ is hypercentral);

(3) $G / C_{G}\left(L_{1}\right)$ is a cyclic group of order dividing $p-1$; and

(4) every subgroup of $\Omega_{1}(P)$ and $\Omega_{n+1}(G) / \Omega_{n}(G)$ is $G$-invariant for each integer $n \geq 1$.

Proof: Let $x \in \Omega_{1}(P)$ so that $\langle x\rangle$ is $G$-invariant by Lemma 1.3. Since $P$ is a locally finite $p$-subgroup, it is locally nilpotent. Then $\langle x\rangle \leq \zeta(P)$. Since this holds for each element of order $p$, we have that $L_{1} \leq \zeta(P)$, that is $P$ is $p$-central. In particular, $L_{1}$ is an elementary abelian $p$-subgroup. Clearly $L_{1}$ is $G$-invariant. By our conditions, every cyclic subgroup of $L_{1}$ is $G$-invariant, and hence every subgroup of $L_{1}$ is $G$-invariant. By 
Lemma 1.6, $G / C_{G}\left(L_{1}\right)$ is cyclic of order a divisor of $p-1$. Pick $g \in G$ such that $G / C_{G}\left(L_{1}\right)=\left\langle g C_{G}\left(L_{1}\right)\right\rangle$.

If $L_{1}=P$, then everything is proved. Suppose therefore that $L_{1} \neq P$ and pick $v \in P$ such that $1 \neq v^{p} \in L_{1}$. Let $F$ be an arbitrary finite subgroup of $G$ such that $g \in f$ and put $V / L_{1}=\langle v\rangle F L_{1} / L_{1}$. Clearly $V / L_{1} \Omega_{1}\left(P / L_{1}\right)=\Omega_{2}(P) / L_{1}$ and so $V / L_{1} \leq \zeta\left(P / L_{1}\right)$ by Corollary 2.6. Consider the mapping

$$
\xi: V \rightarrow L_{1} \text { given by } \xi(y)=y^{p}, \quad y \in V .
$$

Let $y, z \in V$. Then $y^{1} z y=z c$ for some element $c \in L_{1}$. Since $L_{1} \leq \zeta(P)$, we have $y^{n} z y^{n}=z c^{n}$. Since $y^{p} \in L_{1}, z=y^{p} z y^{p}=z c^{p}$ and then $c^{p}=1$. Furthermore, it is not hard to show that

$$
(y z)^{n}=y^{n} z^{n} c^{b_{n}}, \text { where } b_{n}=\frac{(n-1) n}{2} .
$$

In particular, $\xi(y z)=y^{p} z^{p}=\xi(y) \xi(z)$ and so $\xi$ is a homomorphism. Clearly, $\operatorname{Im} \xi \leq L_{1}$ and $\operatorname{Ker} \xi=L_{1}$. Moreover, for each $x \in F$ and $y \in V$, we have

$$
\xi\left(y^{x}\right)=\left(y^{x}\right)^{p}=\left(y^{p}\right)^{x}=\xi(y)^{x} .
$$

Since every subgroup of $L_{1}$ is $F$-invariant, it follows $\xi(y)^{x}=\xi(y)^{t}$, for some positive integer $t$. Then $\xi\left(y^{x}\right)=\xi(y)^{t}=\xi\left(y^{t}\right)$ and hence $y^{x} y^{t} \in$ $\operatorname{Ker} \xi=L_{1}$. Thus

$$
\left(y L_{1}\right)^{x L_{1}}=y^{t} L_{1}=\left(y L_{1}\right)^{t},
$$

which means that $\left\langle y L_{1}\right\rangle$ is $F$-invariant. Moreover, if $x \in C_{F}\left(L_{1}\right)$, then $t=1$ and $\left(y L_{1}\right)^{x L_{1}}=y L_{1}$. Therefore $V / L_{1}=\langle v\rangle F L_{1} / L_{1}=\langle v\rangle L_{1} / L_{1}=$ $\left\langle v L_{1}\right\rangle$. Since this holds for each finite subgroup $F$ containing $g$, every cyclic subgroup of $\Omega_{2}(P) / L_{1}$ is $G$-invariant and so the factor-group $\Omega_{2}(P) / L_{1}$ is $C_{G}\left(L_{1}\right)$-central. It suffices to proceed as above and apply induction to have the required result.

Proposition 2.8. Let $G$ be a locally finite group and $P$ be a normal Sylow p-subgroup of $G$, where $p$ is an odd prime. Suppose that every subgroup of $P$ of prime order is transitively normal in $G$. If $G \neq C_{G}\left(\Omega_{1}(P)\right)$, then the following conditions hold:

(1) $P$ is abelian;

(2) $G / C_{G}(P)$ is a cyclic group of order dividing $p-1$; and

(3) every subgroup of $P$ is $G$-invariant.

Proof: Pick $x \in P$ of order $p$, and let $F$ be a finite subgroup of $P$ containing $x$. Then $\langle x\rangle$ is subnormal in $F$. Being transitively normal, $\langle x\rangle$ is normal in $F$. Since it is true for every finite subgroup of $P$ containing $x$, $\langle x\rangle$ is normal in $P$. It follows that $\langle x\rangle$ is subnormal in $G$, so that $\langle x\rangle$ is 
$G$-invariant. Let $L_{1}=\Omega_{1}(P)$. We have that $L_{1}$ is an elementary abelian $p$-subgroup and $L_{1} \leq \zeta(P)$. By Lemma 1.6, $G / C_{G}\left(L_{1}\right)$ is cyclic of order dividing $p-1$. Let $y \in G$ such that $G / C_{G}\left(L_{1}\right)=\left\langle y C_{G}\left(L_{1}\right)\right\rangle$. Then $y=g h$, where $g$ is a $p^{\prime}$-element, $h$ is a $p$-element and $[g, h]=1$. Since $p$ does not divide $\left|G / C_{G}\left(L_{1}\right)\right|, G / C_{G}\left(L_{1}\right)=\left\langle g C_{G}\left(L_{1}\right)\right\rangle$.

If $L_{1}=P$, then everything is proved. Suppose therefore that $L_{1} \neq P$ and pick $v L_{1} \in \Omega_{1}\left(P / L_{1}\right)=\Omega_{2}(P) / L_{1}$. By Lemma 2.7, $V / L_{1}=\left\langle v L_{1}\right\rangle$ is $G$-invariant. Since $L_{1} \leq \zeta(P), V$ is abelian. Since $L_{1}$ is elementary abelian, $L_{1}=\left\langle v^{p}\right\rangle \times B$, for some subgroup $B$. It follows that $V=\langle v\rangle \times B$. We recall that $B$ is a $G$-invariant subgroup. Since $g$ is a $p^{\prime}$-element, there exists a $\langle g\rangle$-invariant subgroup $U$ such that $V=U \times B$ (see $[\mathbf{1 3}$, Corollary 5.13] for example). Since $U \cong V / B \cong\langle v\rangle, U$ is cyclic, that is $U=\langle u\rangle$ for some element $u \in V$. Then $u^{g}=u^{k_{1}}$, where $k_{1}$ divides $|g|$, and so $p$ does not divide $k_{1}$. Since $|u|=p^{2}$, without loss of generality we can assume that $k_{1}<p^{2}$. By Lemma 1.6, there exists a positive integer $k$ such that $x^{g}=x^{k}$ for every $x \in L_{1}$. Since $L^{p}=\langle 1\rangle$, we can assume that $k<p$. Then

$$
\left(u^{p}\right)^{g}=\left(u^{p}\right)^{k} \text { and }\left(u^{p}\right)^{g}=\left(u^{g}\right)^{p}=\left(u^{k_{1}}\right)^{p}=u^{k_{1} p}=u^{p k_{1}}=\left(u^{p}\right)^{k_{1}} .
$$

It follows that $k_{1} \equiv k(\bmod p)$. Then $k_{1}-k=s p$, for some $s \geq 0$, and thus $k_{1}=k+s p$. We have now $v=u^{t} z$ for some positive integer $t$ and $z \in L_{1}$. Then

$$
v^{g}=\left(u^{t} z\right)^{g}=\left(u^{t}\right)^{g} z^{g}=\left(u^{g}\right)^{t} z^{g}=\left(u^{k_{1}}\right)^{t} z^{k}=\left(u^{t}\right)^{k_{1}} z^{k} .
$$

Further

$$
z^{k_{1}}=z^{k+s p}=z^{k} z^{s p}=z^{k}\left(z^{p}\right)^{s}=z^{k},
$$

because $z^{p}=1$. Thus

$$
v^{g}=\left(u^{t}\right)^{k_{1}} z^{k}=\left(u^{t}\right)^{k_{1}} z^{k_{1}}=\left(u^{t} z^{k}\right)^{k_{1}}=v^{k_{1}} .
$$

Hence every subgroup of $\Omega_{2}(P)$ is $\langle g\rangle$-invariant. Let $F$ be an arbitrary finite subgroup of $\Omega_{2}(P)$. Then $F$ is $\langle g\rangle$-invariant and moreover $g$ induces on $F$ a non-identity power automorphism. It follows that $F$ is abelian by $[\mathbf{1 0}$, Hilfsatz 5$]$. Hence $\Omega_{2}(P)$ is abelian.

Proceeding in this way, we see that every subgroup of $\Omega_{3}(P) / \Omega_{1}(P)$ is $\langle g\rangle$-invariant and then $\Omega_{3}(P) / \Omega_{1}(P)$ is abelian. Pick $w \in \Omega_{3}(P)$ and put $W=\left\langle w, \Omega_{1}(P)\right\rangle$ so that $W$ is abelian since $\Omega_{1}(P) \leq \zeta(P)$. Proceeding as above, we obtain that $\langle w\rangle$ is $\langle g\rangle$-invariant. Applying [10, Hilfsatz 5] again, we obtain that $\Omega_{3}(P)$ is abelian. Applying induction and the above arguments, we see that $\Omega_{n}(P)$ is abelian for each positive integer $n$. Therefore $P=\bigcup_{n \geq 1} \Omega_{n}(P)$ is abelian. 
It follows that $P \leq C_{G}(P)$. Since $P$ is a Sylow $p$-subgroup of $G$ and $G \neq C_{G}(P)$, we have $p \notin \Pi\left(G / C_{G}(P)\right)$. Since the mapping $a \rightarrow$ $a^{p}, a \in \Omega_{2}(P)$ is a $\mathbb{Z} G$-endomorphism of the abelian group $\Omega_{2}(P)$, the factor-group $\Omega_{2}(P) / \Omega_{1}(P)$ is $C_{G}\left(\Omega_{1}(P)\right)$-central. Hence if $a \in \Omega_{2}(P)$ and $y \in C_{G}\left(\Omega_{1}(P)\right)$, then $a^{y}=a c$, for some $c \in \Omega_{1}(P)$. It follows that $y^{n} a y^{n}=a c^{n}$ for every $n \geq 1$. In particular, $y^{p} a y^{p}=a c^{p}=a$, that is $y^{p} \in C_{G}\left(\Omega_{2}(P)\right)$. Since $P$ is abelian, $P \leq C_{G}\left(\Omega_{2}(P)\right)$ and so $G / C_{G}\left(\Omega_{2}(P)\right)$ is a $p^{\prime}$-group. Then the inclusion $y^{p} \in C_{G}\left(\Omega_{2}(P)\right)$ yields $y \in C_{G}\left(\Omega_{2}(P)\right)$. Therefore $C_{G}\left(\Omega_{2}(P)\right)=C_{G}\left(\Omega_{1}(P)\right)$. Proceeding in this way and applying induction, we obtain that $C_{G}\left(\Omega_{n}(P)\right)=C_{G}\left(\Omega_{1}(P)\right)$, for every $n \geq 1$. Hence $C_{G}(P)=C_{G}\left(\Omega_{1}(P)\right)$.

As we proved above, every subgroup of $P$ is $\langle g\rangle$-invariant. Since $G=$ $\langle g\rangle C_{G}(P)$, every subgroup of $P$ is $\langle g\rangle$-invariant.

Lemma 2.9. Let $p$ be an odd prime and let $P$ be a finite $p$-subgroup of a locally finite group $G$. Suppose that $P$ is transitively normal in $G$. If $Q$ is a normal $p^{\prime}$-subgroup of $G$, then $P Q / Q$ is transitively normal in $G / Q$.

Proof: Suppose that $P Q / Q$ is normal in $R / Q$ and $R / Q$ is normal in $T / Q$. Let $x \in T \backslash R$. Since the family $\left\{(P Q / Q)^{y Q} \mid y \in\langle x\rangle\right\}$ is finite and $G$ is locally finite, $D / Q=(P Q / Q)^{\langle x Q\rangle}$ is finite. By the choice of $x$, we have that every conjugate $(P Q / Q)^{y Q} \leq R / Q$ and then it is normal in $R / Q$. It follows that $D / Q$ is a finite $p$-subgroup of $R / Q$. Then $D=Q \lambda S$, where $S$ is a Sylow $p$-subgroup of $D$ (see [7, Theorem 2.4.5] for example). Since the Sylow $p$-subgroups of $D$ are finite, they are conjugate. Therefore there is no loss if we assume that $P \leq S$. Being a finite $p$-subgroup, $S$ is nilpotent. By Lemma 1.3, $P$ is normal in $S$.

The choice of $D / Q$ yields that it is $\langle x Q\rangle$-invariant. Then $D / Q$ is a normal subgroup of $V / Q=D / Q\langle x Q\rangle$. Since the Sylow $p$-subgroups of $D$ are conjugate, they are pronormal in $V$ and it follows that $V=$ $D N_{V}(S)$. Then we may express $x=y z$, where $y \in D$ and $z \in N_{V}(S)$. By Lemma 1.3 again, $P$ is normal in $N_{V}(S)$ and so $P^{z}=P$ and $(P Q / Q)^{z Q}=P Q / Q$. We have that $y=w u$, where $w \in S$ and $u \in Q$. Since $P$ is normal in $S, P^{w}=P$ and $(P Q / Q)^{w Q}=P Q / Q$. Therefore

$$
\begin{aligned}
(P Q / Q)^{x Q}=(P Q / Q)^{w u z Q}=\left(P^{w} Q / Q\right)^{u z Q} & \\
& =(P Q / Q)^{u Q z Q}=(P Q / Q)^{z Q}=P^{z} Q / Q=P Q / Q,
\end{aligned}
$$

and hence $P Q / Q$ is normal in $T / Q$. This gives that $P Q / Q$ is transitively normal in $G / Q$, as required. 
Lemma 2.10. Let $p$ be an odd prime, and let $P$ be an abelian p-subgroup of a locally finite group $G$. Suppose that there exists a $p^{\prime}$-element $x$ such that $x \in N_{G}(P)$ and $[P, x] \neq\langle 1\rangle$. If every subgroup of $P$ of order $p$ is transitively normal in $G$, then every subgroup of $P$ is $\langle x\rangle$-invariant, $P=[P, x]$ and $C_{P}(x)=\langle 1\rangle$.

Proof: By [3, Proposition 2.12], $P=[P, x] \times C_{P}(x)$. Let $H=\langle P, x\rangle$. If we suppose that $H=C_{H}\left(\Omega_{1}(P)\right)$, then $H=C_{H}\left(\Omega_{n}(P)\right)$ for every $n \geq 1$. It follows that $H$ is hypercentral. Since $P$ is a Sylow $p$-subgroup of $H$, $H=P \times\langle x\rangle$ and we obtain a contradiction with the choice of $x$. This contradiction shows that $H \neq C_{H}\left(\Omega_{1}(P)\right)$. By Proposition 2.8, every subgroup of $P$ is $\langle x\rangle$-invariant.

Suppose that $C_{P}(x) \neq\langle 1\rangle$ and pick $c \in C_{P}(x)$ of order $p$. Let now $a \in[P, x]$ of order $p$. Since $a \notin C_{P}(x), a^{x}=a^{d}$, where $d$ is a $p^{\prime}$-number such that $d \not \equiv 1(\bmod p)$. We have

$$
(a c)^{x}=a^{x} c^{x}=a^{x} c .
$$

On the other hand, since $a c \notin C_{P}(x)$,

$$
(a c)^{x}=(a c)^{t},
$$

where $t$ is another $p^{\prime}$-number such that $t \not \equiv 1(\bmod p)$. Hence

$$
a^{d} c=(a c)^{t}=a^{t} c^{t},
$$

and therefore $d \equiv t(\bmod p)$ and $t \equiv 1(\bmod p)$, a contradiction This contradiction proves that $C_{P}(x)=\langle 1\rangle$ and hence $[P, x]=P$.

Lemma 2.11. Let $G$ be a locally finite 2-group. Suppose that every cyclic subgroup of order 2 and of order 4 is transitively normal. Then $G$ is hypercentral.

Proof: Put $L=\Omega_{2}(G)$. If $L \leq \zeta(G)$, then it suffices to apply Corollary 2.6. Therefore suppose that $L$ contains a non-central element $x$. Clearly $|x|=4$. Let $C=C_{G}(\langle x\rangle)$ so that $|G / C|=2$. We claim that $\Omega_{2}(C) \leq \zeta(C)$. For, otherwise there exists $y \in C \backslash \zeta(C)$. Again $|y|=4$ and there exists some $v \in C$ such that $y^{v}=y^{-1}$. We have now $(x y)^{v}=x^{v} y^{v}=x y^{-1}$. Since $|y|=4, x y \neq x y^{-1}$. It follows that the element $x y$ has order 4 , and hence the subgroup $\langle x y\rangle$ is normal in $G$. Then either $(x y)^{v}=x y$ or $(x y)^{v}=(x y)^{-1}=y^{-1} x^{-1}=x^{-1} y^{-1}$, because $y \in C$. In both cases we obtain a contradiction with $(x y)^{v}=x y^{-1}$. Thus $\Omega_{2}(C) \leq \zeta(C)$ as claimed. By Corollary 2.6, $\Omega_{2 n+2}(C) / \Omega_{2 n}(C) \leq$ $\zeta\left(C / \Omega_{2 n}(G)\right)$ for every $n \geq 1$. In particular, $C$ has an ascending series 
of $G$-invariant subgroups

$$
\langle 1\rangle=Z_{0} \leq Z_{1} \leq \cdots \leq Z_{n} \leq Z_{n+1} \leq \cdots \leq C=\bigcup_{n \geq 1} Z_{n}
$$

whose factors are central in $C$ and elementary abelian. Then

$$
\left|G / C_{G}\left(Z_{n+1} / Z_{n}\right)\right| \leq 2
$$

and so the second hypercenter of $G / Z_{n}$ contains $Z_{n+1} / Z_{n}$. Since this holds for every $n \geq 1$, the upper $\omega^{t h}$-hypercenter of $G$ contains $C$. Since $|G / C|=2, G$ is hypercentral.

We are in a position to show Theorem A, whose proof is deduced from the next result.

Theorem 2.12. Let $G$ be a locally finite group whose cyclic subgroups of prime order and of order 4 are transitively normal. Then $G$ satisfies the following conditions:

(1) $G$ is hypercyclic;

(2) the locally nilpotent residual $L$ of $G$ is abelian;

(3) $2 \notin \Pi(L)$ and $\Pi(L) \cap \Pi(G / L)=\emptyset$;

(4) every subgroup of $L$ is $G$-invariant; and

(5) $G / L$ is hypercentral and $G / C_{G}(L)$ is abelian.

Proof: By Corollary 2.3, $L$ is locally nilpotent. Hence

$$
L=\operatorname{Dr}_{p \in \Pi(L)} L_{p},
$$

where $L_{p}$ is the Sylow $p$-subgroup of $L$. We recall that $2 \notin \Pi(L)$ by Corollary 2.4. By Lemma 2.7, each $L_{p}$ has an ascending series of $G$-invariant subgroups whose factors are cyclic. It follows that $L$ has an ascending series of $G$-invariant subgroups whose factors are cyclic.

Therefore it only remains to show that $\Pi(L) \cap \Pi(G / L)=\emptyset$. Suppose that this is false and pick $p \in \Pi(L) \cap \Pi(G / L)$. Clearly, $p$ has to be odd. Since $G$ is locally finite, $G / L$ is locally nilpotent. Hence $G / L=$ $\operatorname{Dr}_{p \in \Pi(G / L)} S_{p}$, where $S_{p}$ is the Sylow $p$-subgroup of $G / L$. In particular, $G / L=P / L \times W / L$, where $P / L=S_{p}$ and $W / L=\operatorname{Dr}_{r \neq p} S_{r}$. We also have $L=R \times Q$, where $R=L_{p}$ and $Q=\operatorname{Dr}_{q \neq p} L_{q}$. Then $P / Q$ is a Sylow $p$-subgroup of $G / Q$. Pick $x \in P$ such that $x^{p} \in Q$. We have $x=u v$, where $u$ is a $p$-element and $v$ is a $p^{\prime}$-element that commute. Then $x Q=u Q$. Since $x^{p} \in Q,|u|=p$ and then the subgroup $\langle u\rangle$ is transitively normal in $G$. By Lemma 2.9, $\langle x Q\rangle=\langle u Q\rangle$ is transitively normal in $G / Q$. In other words, the cyclic subgroups of $G / Q$ of order $p$ are transitively normal in $G / Q$. Denote by $K / Q$ the subgroup of $P / Q$ 
generated by its elements of order $p$. By Lemma 2.7, $K / Q \leq \zeta(P / Q)$, and so $K / Q$ is elementary abelian. Since $P / Q$ is normal in $G / Q$, every cyclic subgroup of $K / Q$ is subnormal in $G / Q$. Being transitively normal, every cyclic subgroup of $K / Q$ is $G$-invariant. Since $K / Q \leq \zeta(P / Q)$, $C_{G / Q}(K / Q) \geq P / Q$. Since $P / Q$ is a Sylow $p$-subgroup of $G / Q$, either $G / Q=C_{G / Q}(K / Q)$ or $(G / Q) / C_{G / Q}(K / Q)$ is a $p^{\prime}$-group. In the first case Lemma 2.7 shows that the factors $\Omega_{n+1}(P / Q) / \Omega_{n}(P / Q)$ are central in $G / Q$ for every $n \geq 1$. It follows that the upper hypercenter of $G / Q$ includes $P / Q$. Since $G / P$ is locally nilpotent, $G / Q$ is locally nilpotent. But $Q$ is a proper subgroup of $L$, and we obtain a contradiction with the choice of $L$. This contradiction shows that $(G / Q) / C_{G / Q}(K / Q)$ is a $p^{\prime}$-group.

By Proposition 2.8, $P / Q$ is abelian, the factor-group $(G / Q) / C_{G / Q}(K / Q)$ is cyclic of order dividing $p-1$ and every subgroup of $P / Q$ is $G$-invariant. Pick $g \in G$ such that $(G / Q) / C_{G / Q}(K / Q)=\left\langle(g Q) C_{G / Q}(K / Q)\right\rangle$. There is no loss if we assume that $g$ is a $p^{\prime}$-element. By our assumption $L / Q \neq P / Q$. Since $G / L$ is locally nilpotent, $\zeta(G / L)$ contains its Sylow $p$-subgroup $\mathrm{P} / \mathrm{L}$, so that $[g Q, P / Q] \leq L / Q$. In particular, $[g Q, P / Q] \neq$ $P / Q$. On the other hand, an application of Lemma 2.10 shows that $[g Q, P / Q]=P / Q$. This contradiction proves that $\Pi(L) \cap \Pi(G / L)=\emptyset$, and (3) is proved.

Let $2 \neq p \in \Pi(G / L), S_{p}$ be a Sylow $p$-subgroup of $G / L$ and $x$ be an element of $G$ such that $x^{p} \in L$. We have express $x=u v$, where $[u, v]=1, u$ is a $p$-element and $v$ is a $p^{\prime}$-element. Then $x L=u L$. Since $x^{p} \in L,|u|=p$. It follows that the subgroup $\langle u\rangle$ is transitively normal in $G$. By Lemma $2.9,\langle x L\rangle=\langle u L\rangle$ is transitively normal in $G / L$. In other words, every cyclic subgroup of $G / L$ having order $p$ is transitively normal in $G / L$. As above we see that all they are $G$-invariant. Moreover, since $G / L$ is locally nilpotent, the center of $G / L$ contains all elements of order $p$. By Corollary 2.6, $\Omega_{n+1}\left(S_{p}\right) / \Omega_{n}\left(S_{p}\right) \leq \zeta\left(S_{p} / \Omega_{n}\left(S_{p}\right)\right)$ for every $n \geq 1$. In particular, $S_{p}$ is hypercentral.

Consider now the Sylow 2-subgroup $S_{2}=E / L$ of $G / L$. Pick $x \in E$ such that $|x L|=2$ or $|x L|=4$, and let $y \in E \backslash L$. Since $G$ is locally finite, $\langle x L, y L\rangle=F / L$ is a finite 2-subgroup. Then $F=L \lambda Y$, where $Y$ is a Sylow 2-subgroup of $F$ (see [7, Theorem 2.4.5] for example). Pick $x_{1}, y_{1} \in Y$ such that $x L=x_{1} L$ and $y L=y_{1} L$. If $|x L|=2$, then $\left|x_{1}\right|=2$. It follows that the subgroup $\left\langle x_{1}\right\rangle$ is transitively normal. By Lemma 1.3, $\left\langle x_{1}\right\rangle$ is normal in $Y$. Since $Y$ is a finite 2 -subgroup, $\left\langle x_{1}\right\rangle \leq \zeta(Y)$. In particular, $x_{1} y_{1}=y_{1} x_{1}$. It follows that

$$
x L y L=x_{1} L y_{1} L=x_{1} y_{1} L=y_{1} x_{1} L=y_{1} L x_{1} L=y L x L .
$$


Since this holds for every element of $E / L, x L \in \zeta(D)$. Suppose now that $|x L|=4$ so that $\left|x_{1}\right|=4$. Again we have that $\left\langle x_{1}\right\rangle$ is transitively normal and then $\left\langle x_{1}\right\rangle$ is normal in $Y$. Thus $y_{1}^{-1} x_{1} y_{1}=x_{1}^{ \pm 1}$. It follows that

$$
(y L)^{-1} x L(y L)=\left(y_{1} L\right)^{-1} x_{1} L\left(y_{1} L\right)=\left(y_{1}^{-1} x_{1} y_{1}\right) L=x_{1}^{ \pm 1} L=(x L)^{ \pm 1} .
$$

Therefore $\langle x L\rangle$ is normal in $E / L$. In other words, every cyclic subgroup of order 2 or order 4 is normal in $S_{2}$. By Lemma 2.11, $S_{2}$ is hypercentral. Since

$G / L$ is hypercentral.

$$
G / L=\operatorname{Dr}_{p \in \Pi(G / L)} S_{p}
$$

Let now $p \in \Pi(L)$ and consider the subgroup $L_{p}$. Since $L$ is locally nilpotent, every cyclic subgroup of $L_{p}$ of prime order is $G$-invariant by Lemma 1.3. In particular, $\Omega_{1}\left(L_{p}\right) \leq \zeta\left(L_{p}\right)$. In other words, $C_{G}\left(\Omega_{1}\left(L_{p}\right)\right) \geq$ $L_{p}$. Then either $G=C_{G}\left(\Omega_{1}\left(L_{p}\right)\right)$ or $G / C_{G}\left(\Omega_{1}\left(L_{p}\right)\right)$ is a $p^{\prime}$-group. In the first case, by Lemma 2.7 , the factors $\Omega_{n+1}\left(L_{p}\right) / \Omega_{n}\left(L_{p}\right)$ are central in $G$ for every $n \geq 1$. It follows that the upper hypercenter of $G$ includes $L_{p}$. Since $G / L$ is locally nilpotent, $G /\left(\operatorname{Dr}_{q \neq p} L_{q}\right)$ is locally nilpotent. On the other hand, $\operatorname{Dr}_{q \neq p} L_{q}$ is a proper subgroup of $L$ and we obtain a contradiction with the choice of $L$. This contradiction shows that $G / C_{G}\left(\Omega_{1}\left(L_{p}\right)\right)$ is a $p^{\prime}$-group. By Proposition $2.8, L_{p}$ is abelian, the factor-group $G / C_{G}\left(\Omega_{1}\left(L_{p}\right)\right)$ is cyclic of order dividing $p-1$ and every subgroup of $L_{p}$ is $G$-invariant. Since this holds for every $p \in \Pi(L), L$ is abelian, the factor-group $G / C_{G}(L)$ is abelian and every subgroup of $L$ is $G$-invariant. The proof is now complete.

The original results due to P. Csörgö and M. Herzog [5] and Li Yangming [18] appear now as immediate consequences of Theorem A.

Corollary $2.13([5])$. Let $G$ be a finite group whose cyclic subgroups of prime order and of order 4 are $\mathcal{H}$-subgroups. Then $G$ satisfies the following conditions:

(1) $G$ is supersoluble;

(2) $G=L \lambda K$, where $L$ and $K$ are nilpotent Hall subgroups of $G$;

(3) every cyclic subgroup of prime order and of order 4 of $L$ is $G$-invariant; and

(4) every cyclic subgroup of prime order and of order 4 of $K$ is normal in $K$.

Corollary $2.14([\mathbf{1 8}])$. Let $G$ be a finite group whose cyclic subgroups of prime order and of order 4 are NE-subgroups. Then $G$ is supersoluble. 
From this, we are able to obtain the following most wide characterization of locally finite $\bar{T}$-groups. The next result is an extended version of Corollary A1.

Corollary 2.15. Let $G$ be a locally finite group whose primary cyclic subgroups are transitively normal. If $L$ is the locally nilpotent residual of $G$, then we have

(1) L is abelian;

(2) $2 \notin \Pi(L)$ and $\Pi(L) \cap \Pi(G / L)=\emptyset$;

(3) every subgroup of $C_{G}(L)$ is $G$-invariant; and

(4) $G / L$ is Dedekind and $G / C_{G}(L)$ is abelian.

In particular, $G$ is a a $\bar{T}$-group.

Proof: Since every subgroup of prime order and every subgroup of order 4 is transitively normal, $G$ satisfies the conditions of Theorem 2.12. The assertions (1)-(3) follow from Theorem 2.12. We only have to prove that the factor-group $G / L$ is a Dedekind group. Let $p \in \Pi(G / L)$ and $P / L$ be a Sylow $p$-subgroup of $G / L$. Pick $x L, y L \in P / L$. Since $G$ is locally finite, $G / L$ is locally nilpotent, so that $\langle x L, y L\rangle=K / L$ is a finite $p$-subgroup. Then there exists a finite subgroup $F$ such that $K=F L$. Let $V$ be a Sylow $p$-subgroup of $F$. Then $V(F \cap L) /(F \cap L)$ is a Sylow $p$-subgroup of $F /(F \cap L)$. Since $F /(F \cap L) \cong F L / L$ is a $p$-group, $V(F \cap L)=F$ and then $V L=K$. By Corollary 1.4, $V$ is a Dedekind group. It follows that $V L / L=K / L$ is also a Dedekind group. Therefore $\langle x L\rangle^{y L}=\langle x L\rangle$. This holds for every element $y L \in P / L$ so that $\langle x L\rangle$ is normal in $P / L$. Hence $P / L$ is a Dedekind group, and so is $G / L$.

Since $G$ satisfies the conditions (1)-(4), $G$ is a $\bar{T}$-group by $[\mathbf{2 2}$, Theorem 6.1.1].

Corollary 2.16. Let $G$ be a locally finite group. Then the following assertions are equivalent:

(1) every primary cyclic subgroup of $G$ is transitively normal;

(2) every primary cyclic subgroup of $G$ is an $\mathcal{H}$-subgroup;

(3) every primary cyclic subgroup of $G$ is an NE-subgroup;

(4) every primary cyclic subgroup of $G$ is weakly normal;

(5) every primary cyclic subgroup of $G$ is pronormal;

(6) every cyclic subgroup of $G$ is pronormal; and

(7) $G$ is a $\bar{T}$-group. 
Proof: The assertion (1) $\Rightarrow(6)$ follows from Theorem B. Conversely, if $G$ is a $\bar{T}$-group, then every cyclic subgroup of $G$ is pronormal ([25]).

We note that if every cyclic subgroup of a finite group $G$ is pronormal, then every subgroup is pronormal $([\mathbf{2 0}])$ and that the class of finite $\bar{T}$-groups coincides with the class of soluble $T$-groups $([\mathbf{8}])$. Therefore for finite groups we obtain.

Corollary 2.17. Let $G$ be a finite group. Then the following assertions are equivalent:

(1) every primary cyclic subgroup of $G$ is transitively normal;

(2) every primary cyclic subgroup of $G$ is an $\mathcal{H}$-subgroup;

(3) every primary cyclic subgroup of $G$ is an $N E$-subgroup;

(4) every primary cyclic subgroup of $G$ is weakly normal;

(5) every primary cyclic subgroup of $G$ is pronormal;

(6) every cyclic subgroup of $G$ is pronormal;

(7) every subgroup of $G$ is pronormal;

(8) $G$ is a $\bar{T}$-group; and

(9) $G$ is a soluble T-group.

This result gives a very wide generalization of results of the papers [4] and $[\mathbf{1 8}]$.

\section{Some non-periodic groups whose cyclic subgroups are transitively normal}

We start with some auxiliary results.

Lemma 3.1. Let $G$ be a group whose cyclic subgroups are transitively normal. If $A \leq G$ is periodic locally nilpotent and $g \in N_{G}(A)$ has infinite order, then $g \in C_{G}(A)$.

Proof: We have $A=\operatorname{Dr}_{p \in \Pi(A)} A_{p}$, where $A_{p}$ is the Sylow $p$-subgroup of $A$. Put $L_{p}=\Omega_{1}\left(A_{p}\right)$ for every $p \in \Pi(A)$. By Lemma 1.3, every cyclic subgroup of $L_{p}$ is $\langle g\rangle$-invariant. Then Lemma 1.6 yields $g^{k} \in C_{G}\left(L_{p}\right)$, where $k$ is a divisor of $p-1$. Since the mapping $a \rightarrow a^{p}, a \in \Omega_{2}\left(A_{p}\right)$ is a $\mathbb{Z}\langle g\rangle$-homomorphism, $g^{k} \in C_{G}\left(\Omega_{2}\left(A_{p}\right) / \Omega_{1}\left(A_{p}\right)\right)$. Similarly, $g^{k} \in$ $C_{G}\left(\Omega_{n+1}\left(A_{p}\right) / \Omega_{n}\left(A_{p}\right)\right)$ for every positive integer $n$. It follows that the subgroup $\left\langle g_{p}, A_{p}\right\rangle$, where $g_{p}=g^{k}$, is hypercentral. By Corollary 1.4, $\left\langle g_{p}, A_{p}\right\rangle$ is a Dedekind group. Being non-periodic, it has to be abelian. Pick $y \in A_{p}$. Then $g_{p} y$ has infinite order. The subgroup $\left\langle g_{p}, A_{p}\right\rangle$ is normal in $\left\langle g, A_{p}\right\rangle$ and $\left\langle g_{p} y\right\rangle$ is normal in $\left\langle g_{p}, A_{p}\right\rangle$. It follows that $\left\langle g_{p} y\right\rangle$ is 
normal in $\left\langle g, A_{p}\right\rangle$. Therefore either $g^{-1}\left(g_{p} y\right) g=\left(g_{p} y\right)^{-1}$ or $g^{-1}\left(g_{p} y\right) g=$ $g_{p} y$. In the first case we have

$$
g_{p}^{-1} y^{-1}=\left(g_{p} y\right)^{-1}=g^{-1}\left(g_{p} y\right) g=\left(g^{-1} g_{p} g\right)\left(g^{-1} y g\right)=g_{p} y^{t},
$$

for some positive integer $t$. Since $\left\langle g_{p}, y\right\rangle=\left\langle g_{p}\right\rangle \times\langle y\rangle$, we obtain that $g_{p}^{-1}=g_{p}$, which is impossible because $g_{p}^{-1}$ has infinite order. This contradiction shows that $g^{-1}\left(g_{p} y\right) g=g_{p} y$. Then

$$
g^{-1} y g=g^{-1}\left(g_{p}^{-1}\left(g_{p} y\right)\right) g=\left(g^{-1} g_{p}^{-1} g\right)\left(g^{-1}\left(g_{p} y\right) g\right)=g_{p}^{-1}\left(g_{p} y\right)=y \text {. }
$$

Since this holds for each element $y \in A_{p}, g \in C_{G}\left(A_{p}\right)$. The last inclusion is valid for all $p \in \Pi(A)$ and then $g \in C_{G}(A)$. Hence $\langle g, A\rangle$ is abelian.

Corollary 3.2. Let $G$ be a group whose cyclic subgroups are transitively normal. Suppose that $A$ is a periodic locally nilpotent subgroup of $G$ and $g \in N_{G}(A)$. If $g$ has infinite order, then $g \in C_{G}(A)$.

Proof: We have $A=\operatorname{Dr}_{p \in \Pi(A)} A_{p}$, where $A_{p}$ is the Sylow $p$-subgroup of $A$. By Corollary 1.4, $A$ is a Dedekind group. Then either $A$ is abelian or $A_{p}$ is abelian for every odd prime $p$ and $A_{2}=Q \times D$, where $Q$ is a quaternion group and $D$ is an elementary abelian 2-subgroup. In the first case, the result follows from Lemma 3.1.

Thus we consider the second case. Applying Lemma 3.1 again, we see that $g \in C_{G}\left(A_{p}\right)$ for every odd prime $p$. Suppose that $g \notin C_{G}\left(A_{2}\right)$. By Lemma 1.3, every subgroup of $A_{2}$ is $\langle g\rangle$-invariant. Since the maximal order of the elements of $A_{2}$ is 4 , it follows that $g^{2} \in C_{G}\left(A_{2}\right)$. Therefore the subgroup $\left\langle g^{2}, A^{2}\right\rangle$ is nilpotent. By Corollary $1.4,\left\langle g^{2}, A^{2}\right\rangle$ is a Dedekind group. Being non-periodic, it has to be abelian and we obtain a contradiction. This contradiction proves the result.

Lemma 3.3. Let $A$ be a non-periodic abelian subgroup of a group $G$ whose cyclic subgroups are transitively normal. If $b \in N_{G}(A) \backslash C_{G}(A)$, then $b^{2} \in C_{G}(A)$ and $a^{b}=a^{-1}$ for every element $a \in A$.

Proof: Let $a \in A$ be arbitrary. Being transitively normal, $\langle a\rangle$ is normal in $\langle a, A\rangle$. If the order of $a$ is infinite, then either $a^{b}=a$ or $a^{b}=a^{-1}$. In other words, $b$ centralizes or inverts each non-periodic element of $A$.

If $a^{b}=a$ for every $a \in A$, then the result follows. Otherwise, there exists $c \in A$ such that $c^{b}=c^{-1} \neq c$. Actually we may suppose that the order of $c$ is infinite. For, if $b$ centralizes every non-periodic element of $A$, this forces that $c$ must have order 2 , a contradiction. In this case, we are going to show that $b$ inverts every element $a \in A$.

Suppose that $a$ has finite order so that $a c$ has infinite order. Then

$$
a^{b} c^{-1}=(a c)^{b}=a c \text { or } a^{-1} c^{-1} .
$$


In the first case we have $c^{2}=a^{b} a^{-1}$, a contradiction since $c^{2}$ has infinite order. It follows that $a^{b}=a^{-1}$. Suppose now that $a$ has infinite order. If $a v$ has finite order, reasoning as above we have

$$
a^{b} c^{-1}=(a c)^{b}=(a c)^{-1}=a^{-1} c^{-1}
$$

hence $a^{b}=a^{-1}$. If $a c$ has infinite order, we apply our claim to $a$ and $a c$ at once to easily see that $b$ cannot centralize $a$. Hence $v^{b}=v^{-1}$, as required.

We are closer to establishing the proof of the necessity of the conditions of Theorem B.

Lemma 3.4. Suppose we have a group $G$ of the form $G=A\langle b\rangle$, where $A$ is a non-periodic abelian normal subgroup of $G, b^{2} \in A$ and $a^{b}=a^{-1}$ for every element $a \in A$. If every cyclic subgroup of $G$ is transitively normal, then we have

(1) if $b^{2}=1$, then the Sylow 2-subgroup $D$ of $A$ is elementary abelian; and

(2) if $b^{2} \neq 1$, then either $D$ is elementary abelian or $D=E \times\langle v\rangle$ where $E$ is elementary abelian and $\langle b, v\rangle$ is a quaternion group.

In both cases $\langle D, b\rangle$ is a Dedekind group.

Proof: Pick $a \in C_{A}(b)$ so that $a^{b}=a$. Since $a^{b}=a^{-1}$ by hypothesis, we have $a^{2}=1$. Therefore $C_{A}(b)$ is an elementary abelian 2-subgroup. Since $b^{2} \in A$, we have $b^{2} \in C_{A}(b)$. If $b^{2} \neq 1$, then $b^{4}=\left(b^{2}\right)^{2}=1$ and so $b$ has order 4 . Conversely, it is trivial that if $a \in A$ and $|a|=2$, then $a \in C_{A}(b)$.

Let $a$ be an arbitrary element of $A$. Then $b^{-1} a^{1} b a=a^{2}$ and so $a^{-1} b a=b a^{2}$. Moreover $a b=b a^{-1}$ and then $(b a)(b a)=b(a b) a=$ $b\left(b a^{-1}\right) a=b^{2}$.

Assume that there exists a subgroup $L \leq G$ such that $b \in L$ and $\langle b\rangle$ is subnormal in $L$. We have $L=(L \cap A)\langle b\rangle$ and $K:=L \cap A$ is an abelian normal subgroup of $L$. Since $\langle b\rangle$ is subnormal in $L, L=K\langle b\rangle$ is nilpotent (see [9, Lemma 4]). Actually $\Pi(K)=\{2\}$. For, otherwise suppose that $x \in K$ is a $2^{\prime}$-element. Since $L$ is nilpotent, $x \in C_{L}(b)$, a contradiction because we have already seen that $C_{A}(b)$ is an elementary abelian 2-subgroup.

Suppose that $y \in K$ has infinite order. Since every subgroup of $A$ is $G$-invariant, the subgroup $\langle y\rangle \cap \zeta(L):=\langle z\rangle$ has to be non-trivial. Again $z \in C_{L}(b)$, which leads to a contradiction. Therefore $K$ is periodic and hence a 2 -subgroup. 
Let $D$ be the Sylow 2-subgroup of $A$ so that $K \leq D$. The subgroup $\langle D, b\rangle$ is a locally nilpotent 2 -group and then $\langle b\rangle$ is normal in $\langle D, b\rangle$ by Lemma 1.3. Moreover, Corollary 1.4 shows that $\langle D, b\rangle$ is a Dedekind group.

Suppose first $|b|=2$. Then $\langle b\rangle \cap A=\langle 1\rangle$. Since $\langle b\rangle$ is normal in $\langle D, b\rangle$, $\langle D, b\rangle=D \times\langle b\rangle$. It follows that $D \leq C_{A}(b)$, an elementary abelian 2 -subgroup. Otherwise $b$ has order 4 . Since $\left\langle b^{2}\right\rangle$ is normal in $G$, reasoning as above, we see that $D /\left\langle b^{2}\right\rangle$ is an elementary abelian 2-subgroup. Then either $D$ is elementary abelian or $D$ has an element $v$ of order 4 such that $v^{2}=b^{2}$. In this case, since the order of $v$ is maximal among the order of the elements of $D$, there exists an elementary abelian subgroup $E$ such that $D=E \times\langle v\rangle$. Since $\langle v\rangle$ is $\langle b\rangle$-invariant,

$$
|\langle b\rangle\langle v\rangle|=(|\langle b\rangle||\langle v\rangle|) /|\langle b\rangle \cap\langle v\rangle|=8 .
$$

Since $\langle b\rangle$ is normal in $\langle D, b\rangle,\langle b, v\rangle$ is a product of two cyclic normal subgroups of order 4 . It readily follows that $\langle v, b\rangle$ is a quaternion group.

Proposition 3.5. Let $G$ be a non-abelian radical group whose cyclic subgroups are transitively normal in $G$. If $G$ is non-periodic, then $G=$ $A\langle b\rangle$, where $A$ is an abelian normal subgroup, $b^{2} \in A$ and $a^{b}=a^{-1}$ for every element $a \in A$. Moreover

(1) if $b^{2}=1$, then the Sylow 2-subgroup $D$ of $A$ is elementary abelian; and

(2) if $b^{2} \neq 1$, then either $D$ is elementary abelian or $D=E \times\langle v\rangle$, where $E$ is elementary abelian and $\langle b, v\rangle$ is the quaternion group.

In both cases $\langle D, b\rangle$ is a Dedekind group.

Proof: Let $L$ be the locally nilpotent radical of $G$ so that $L$ itself is locally nilpotent. We claim that $L$ cannot be periodic. For, otherwise suppose that $L$ is periodic. Pick an element $g \in G$ of infinite order. By Corollary 3.2, $g \in C_{G}(L)$. On the other hand, being $G$ radical, we have that $C_{G}(L) \leq L([\mathbf{2 1}$, Lemma 4$])$, a contradiction, which shows our claim.

By Corollary 1.4, $L$ is a non-periodic Dedekind group and hence abelian. By Corollary 1.5, every subgroup of $L$ is $G$-invariant. It follows that $G / C_{G}(L)$ is a finite group of order 2 (see [23, Theorem 1.5.7]). Since $C_{G}(L) \leq L$, there exists some $b \in G$ such that $G=L\langle b\rangle$ and $b^{2} \in L$. Put $A=L$. It suffices to apply Lemma 3.3 and Lemma 3.4 to get the result.

To show the aforesaid necessity in Theorem B, it only remains to show that the groups in its statement actually are radical. 
Corollary 3.6. A non-periodic non-abelian locally radical group $G$ whose cyclic subgroups are transitively normal is radical.

Proof: Pick elements $g, x, y \in G$ such that the order of $g$ is infinite and $x y \neq y x$. Put $F=\langle g, x, y\rangle$ and let $\mathcal{L}$ be the local system of $G$ consisting of all finitely generated subgroups of $G$ that contains $F$. By Proposition 3.5, every subgroup $K \in \mathcal{L}$ is metabelian. It follows that $G$ itself is metabelian hence radical.

In a famous paper I. Schur $[\mathbf{2 4}]$ proved the following outstanding result: If the factor-group $G / \zeta(G)$ is finite, then the commutator subgroup $[G, G]$ is finite. From this it is not hard to deduce as a corollary the following slight generalization: If the factor-group $G / \zeta(G)$ is locally finite, then the commutator subgroup $[G, G]$ is locally finite.

Lemma 3.7. A non-periodic generalized radical group $G$ whose cyclic subgroups are transitively normal is a radical group.

Proof: Let $L$ be the locally nilpotent radical of $G$. We claim that $L \neq$ $\langle 1\rangle$; this is clear if $G$ has no non-trivial locally finite normal subgroups since $G$ is generalized radical. Otherwise, if $F$ is the locally finite radical of $G$, we have that $F \neq 1$. By Corollary 2.15, $[F, F]$ is abelian and thus $L$ is non-trivial just as we claimed.

Assume that $L$ contains no $C_{G}(L)$ and set $K:=L C_{G}(L)$ so that $K / L \neq\langle 1\rangle$. Since $G$ is generalized radical, there exists an ascending series of $G$-invariant subgroups

$$
L=K_{0} \leq K_{1} \leq \cdots \leq K_{\eta}=K,
$$

whose factors are either locally nilpotent or locally finite. We recall that the locally nilpotent radical of a radical group contains its centralizer ([21, Lemma 4]). It follows that the factor $K_{1} / L$ is locally finite since it cannot be locally nilpotent. If $L$ is periodic, then $K_{1}$ itself is locally finite and then, by Corollary 2.15, $K_{1} / L$ is a Dedekind group, a contradiction. Hence $L$ is not periodic. By Corollary 1.4, $L$ is also a Dedekind group and hence it has to be abelian. Put $C=C_{G}(L) \cap L$ and $D=C_{G}(L) \cap K_{1}$. Then $C \leq \zeta(D)$ and

$$
D / C=\left(C_{G}(L) \cap K_{1}\right) /\left(C_{G}(L) \cap K_{1} \cap L\right) \cong\left(C_{G}(L) \cap K_{1}\right) L / L \leq K_{1} / L .
$$

Since $K_{1} / L$ contains no locally nilpotent normal subgroups, $D / C$ is locally finite and contains no locally nilpotent normal subgroups. As mentioned above, $[D, D]$ is locally finite. By Corollary $2.15,[D, D]$ is metabelian and then $D$ is soluble. Hence $D / C$ is soluble and this leads to a contradiction. Consequently $C_{G}(L) \leq L$ and then $G$ is a radical group. 
Corollary 3.8. A non-periodic non-abelian locally generalized radical group whose cyclic subgroup are transitively normal is radical.

Proof of Theorem B: The structure of $G$ follows from the application of the previous results.

Suppose that a non-periodic non-abelian locally generalized radical group $G$ has the structure given in the statement. Let $g \in G$ be an arbitrary element. If $g \in L$, then $\langle g\rangle$ is normal in $G$ and hence it is transitively normal. Suppose that $g \notin L$. Then there exists some $u \in L$ such that $g=b u$. Therefore we have $G=L\langle g\rangle$. Pick $a \in L$. Then

$$
b^{-1} a^{-1} b a=a a=a^{2}
$$

and so $a^{-1} b a=b a^{2}$. Since $a b=b a^{-1}$,

$$
(b a)^{2}=b(a b) a=b\left(b a^{-1}\right) a=b^{2} .
$$

In particular, $g^{2}=(b u)^{2}=b^{2}$. Since $L$ is abelian, $a^{g}=a^{b}=a^{-1}$ for every element $a \leq L$, which assures us that it will suffice to show that $\langle b\rangle$ is transitively normal in $G$.

Let $K$ be a subgroup of $G$ such that $\langle b\rangle$ is subnormal in $K$ and put $F=\langle b\rangle^{K}$. Then $F$ is a 2-subgroup. If $K$ itself is a 2-subgroup, then $K$ is a Dedekind group, so that $\langle b\rangle$ is normal in $K$. Suppose first that there exists $y \in K$ such that $|y|=p$ is an odd prime. Then $y \in L$. As we proved above, $y^{-1} b y=b y^{2}$ and so $y^{2} \in F$. On the other hand, by the choice of $y$ we have that $\langle y\rangle=\left\langle y^{2}\right\rangle$ and then $y \in E$, a contradiction since $F$ is a 2-subgroup. This contradiction shows that $\Pi(K)=\{2\}$ if $K$ has elements of order finite. Otherwise, pick $d \in K$ of infinite order. Reasoning as above we obtain that $d^{2} \in F$, a new contradiction since $F$ is periodic and $d^{2}$ is not. Thus $K$ has to be a 2 -subgroup and the result follows.

Acknowledgements. This paper is supported by Proyecto MTM201019938-C03-03 of the Department of I+D+i of MINECO (Spain), the Department of I+D of the Government of Aragón (Spain) and FEDER funds from European Union. The authors also wish to thank the valuable comments of the referees that have allowed to improve very much the contents of the paper.

\section{References}

[1] R. BAER, Situation der Untergruppen und Struktur der Gruppe, Sitzungsber. Heidelb. Akad. Wiss. 2 (1933), 12-17. 
[2] A. Ballester-Bolinches and R. Esteban-Romero, On finite T-groups, J. Aust. Math. Soc. 75(2) (2003), 181-191. DOI: 10.1017/S1446788700003712.

[3] A. Ballester-Bolinches, L. A. Kurdachenko, J. Otal, and T. Pedraza, Infinite groups with many permutable subgroups, Rev. Mat. Iberoam. 24(3) (2008), 745-764. DOI: 10.4171/RMI/555.

[4] M. Bianchi, A. G. B. Mauri, M. Herzog, and L. Verardi, On finite solvable groups in which normality is a transitive relation, J. Group Theory 3(2) (2000), 147-56. DOI: 10.1515/jgth.2000.012.

[5] P. Csörgö And M. Herzog, On supersolvable groups and the nilpotator, Comm. Algebra 32(2) (2004), 609-620. DOI: 10.1081/ AGB-120027916.

[6] R. Dedekind, Ueber Gruppen, deren sämmtliche Theiler Normaltheiler sind, Math. Ann. 48(4) (1897), 548-561. DOI: 10.1007/ BF01447922.

[7] M. R. Dixon, "Sylow theory, formations and Fitting classes in locally finite groups", Series in Algebra 2, World Scientific Publishing Co., Inc., River Edge, NJ, 1994.

[8] W. Gaschütz, Gruppen, in denen das Normalteilersein transitiv ist, J. Reine Angew. Math. 198 (1957), 87-92. DOI: 10.1515/crll. 1957.198.87.

[9] H. Heineken and L. A. Kurdachenko, Groups with subnormality for all subgroups that are not finitely generated, Ann. Mat. Pura Appl. (4) 169 (1995), 203-232. DOI: 10.1007/BF01759354.

[10] B. Huppert, "Endliche Gruppen. I", Die Grundlehren der mathematischen Wissenschaften 134, Springer-Verlag, Berlin-New York, 1967.

[11] M. I. Kargapolov, On generalized solvable groups, (Russian), Algebra i Logika Sem. 2(5) (1963), 19-28.

[12] L. G. Kovács, B. H. Neumann, And H. De Vries, Some Sylow subgroups, Proc. Roy. Soc. Ser. A 260 (1961), 304-316.

[13] L. A. Kurdachenko, J. Otal, and I. Ya. Subbotin, "Artinian modules over group rings", Frontiers in Mathematics, Birkhäuser Verlag, Basel, 2007.

[14] L. A. Kurdachenko and I. Ya. Subbotin, Transitivity of normality and pronormal subgroups, in: "Combinatorial group theory, discrete groups, and number theory", Contemp. Math. 421, Amer. Math. Soc., Providence, RI, 2006, pp. 201-212. DOI: 10.1090/conm/ $421 / 08038$.

[15] T. J. LAFFEY, A lemma on finite $p$-groups and some consequences, Proc. Cambridge Philos. Soc. 75 (1974), 133-137. 
[16] S. Li, On minimal subgroups of finite groups, Comm. Algebra 22(6) (1994), 1913-1918. DOI : 10.1080/00927879408824946.

[17] S. Li, On minimal non-PE-groups, J. Pure Appl. Algebra 132(2) (1998), 149-158. DOI : 10.1016/S0022-4049(97)00106-0.

[18] Y. LI, Finite groups with $N E$-subgroups, J. Group Theory 9(1) (2006), 49-58. DOI: 10.1515/JGT.2006.003.

[19] K. H. MülLER, Schwachnormale Untergruppen: Eine gemeinsame Verallgemeinerung der normalen und normalisatorgleichen Untergruppen, Rend. Sem. Mat. Univ. Padova 36 (1966), 129-157.

[20] T. A. Peng, Finite groups with pro-normal subgroups, Proc. Amer. Math. Soc. 20 (1969), 232-234. DOI: 10.1090/S0002-9939-19690232850-1.

[21] B. I. Plotkin, Radical groups, (Russian), Mat. Sb. N.S. 37(79) (1955), 507-526.

[22] D. J. S. Robinson, Groups in which normality is a transitive relation, Proc. Cambridge Philos. Soc. 60 (1964), 21-38.

[23] R. Schmidt, "Subgroup lattices of groups", de Gruyter Expositions in Mathematics 14, Walter de Gruyter \& Co., Berlin, 1994. DOI: 10.1515/9783110868647.

[24] I. SchuR, Über die Darstellungen der endlichen Gruppen durch gebrochene lineare Substitutionen, J. reine angew. Math. 127 (1904), 20-50.

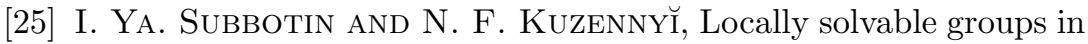
which all infinite subgroups are pronormal, (Russian), Izv. Vyssh. Uchebn. Zaved. Mat. 11 (1988), 77-79; translation in: Soviet Math. (Iz. VUZ) 32(11) (1988), 126-131.

Leonid A. Kurdachenko:

Department of Algebra

National University of Dnepropetrovsk

Dnepropetrovsk

Ukraine 49010

E-mail address: 1kurdachenko@i.ua

Javier Otal:

Department of Mathematics - IUMA

University of Zaragoza

50009 Zaragoza

Spain

E-mail address: otal@unizar.es

Primera versió rebuda el 31 d'octubre de 2011, darrera versió rebuda el 23 de juliol de 2012. 